\title{
The INTEGRAL ground segment and its science operations centre
}

\author{
R. Much ${ }^{1}$, P. Barr ${ }^{1}$, L. Hansson ${ }^{1}$, E. Kuulkers ${ }^{1}$, P. Maldari ${ }^{2}$, J. Nolan ${ }^{1}$, T. Oosterbroek ${ }^{1}$, A. Orr ${ }^{1}$, \\ A. N. Parmar ${ }^{1}$, M. Schmidt ${ }^{2}$, J. Sternberg ${ }^{1}$, O. R. Williams ${ }^{1}$, and C. Winkler ${ }^{1}$ \\ ${ }^{1}$ Research and Scientific Support Department of ESA, ESTEC, Keplerlaan 1, 2201 AZ Noordwijk, The Netherlands \\ 2 ESA-ESOC, Mission Operations Department, Robert-Bosch Str. 5, 64293 Darmstadt, Germany
}

Received 2 July 2003 / Accepted 14 August 2003

\begin{abstract}
The INTEGRAL ground segment is divided into operational and scientific components. The operational component consists of the Mission Operations Centre, the ground stations and communications lines while the scientific component comprises of a Science Operations Centre and Science Data Centre. The overall architecture of the ground segment is described paying particular attention to the tasks and functionalities of the INTEGRAL Science Operations Centre.
\end{abstract}

Key words. miscellaneous

\section{Introduction}

The INTEGRAL Ground Segment is divided into operational and science components and the overall structure and interfaces are illustrated in Fig. 1. The Operational Ground Segment comprises of the INTEGRAL Mission Operations Centre (MOC) located at ESOC in Darmstadt (Germany), two ground stations and the communications system. The INTEGRAL Science Operations Centre (ISOC) located at ESTEC in Noordwijk (The Netherlands) and the INTEGRAL Science Data Centre (ISDC) located at Versoix (Switzerland) together form the Science Ground Segment. The scientific community interacts directly with both components of the Science Ground segment. The two segments are described in more detail below.

\section{The INTEGRAL operational ground segment}

The MOC is responsible for the operation of the spacecraft and instruments, for ensuring the spacecraft safety and health, for the maintenance of the satellite's on-board software, for the provision of flight dynamics support including determination and control of the satellite's orbit and attitude and for provision of the telemetry and auxiliary data to the ISDC. The MOC is the sole interface to the spacecraft and all commands to be sent to the spacecraft or instruments are generated at the MOC.

INTEGRAL operations are executed using an automated timeline. The timeline is generated on a revolution by revolution basis, i.e. one timeline covers the period from perigee passage to the next perigee passage, which is approximately three days. Although there are already activities much before (the planning of ground station resources commences a

Send offprint requests to: $\mathrm{R}$. Much, e-mail: Rudolf.Much@rssd.esa.int year before), the actual planning process starts when the MOC sends Planning Skeleton Files to the ISOC about one month before observation execution. The Planning Skeleton Files define the time intervals reserved for dedicated spacecraft operation, instrument activation and de-activation, handovers between the ground stations and for scientific observations. Using these as input, the ISOC generates Planned Observation Sequence (POS) files which are then sent to the MOC. POS files contain the times when the astronomical targets selected for a particular revolution will be observed, the target pointings and instrument configurations, together with time windows for other necessary spacecraft activities. The time at which an approved target will be observed by INTEGRAL depends on INTEGRAL's celestial viewing constraints, on whether it is a fixed time observation and on the overall efficiency at which it fits into the observing programme. After a POS has been received by the MOC its correctness is verified and the high-level information is converted into command sequences and parameter settings which are retrieved from an operational data base, which is under configuration control. Finally, an operational timeline is generated and a summary is sent to the ISOC for confirmation. The approved command schedule is then loaded into the command system at MOC during the ground station outage at the satellite's perigee passage before the orbital revolution concerned. The commands are then executed automatically by the command system, and manual commanding is only envisaged under special circumstances such as contingencies.

Since INTEGRAL is controlled in real-time from the ground, a network of stations has been implemented which allows permanent contact with the satellite during the scientifically useful part of the orbit. This consists of two ground stations, one located at Redu in Belgium (provided by ESA), and one at Goldstone in California, USA (provided by NASA/Jet Propulsion Laboratory). The MOC receives all satellite 


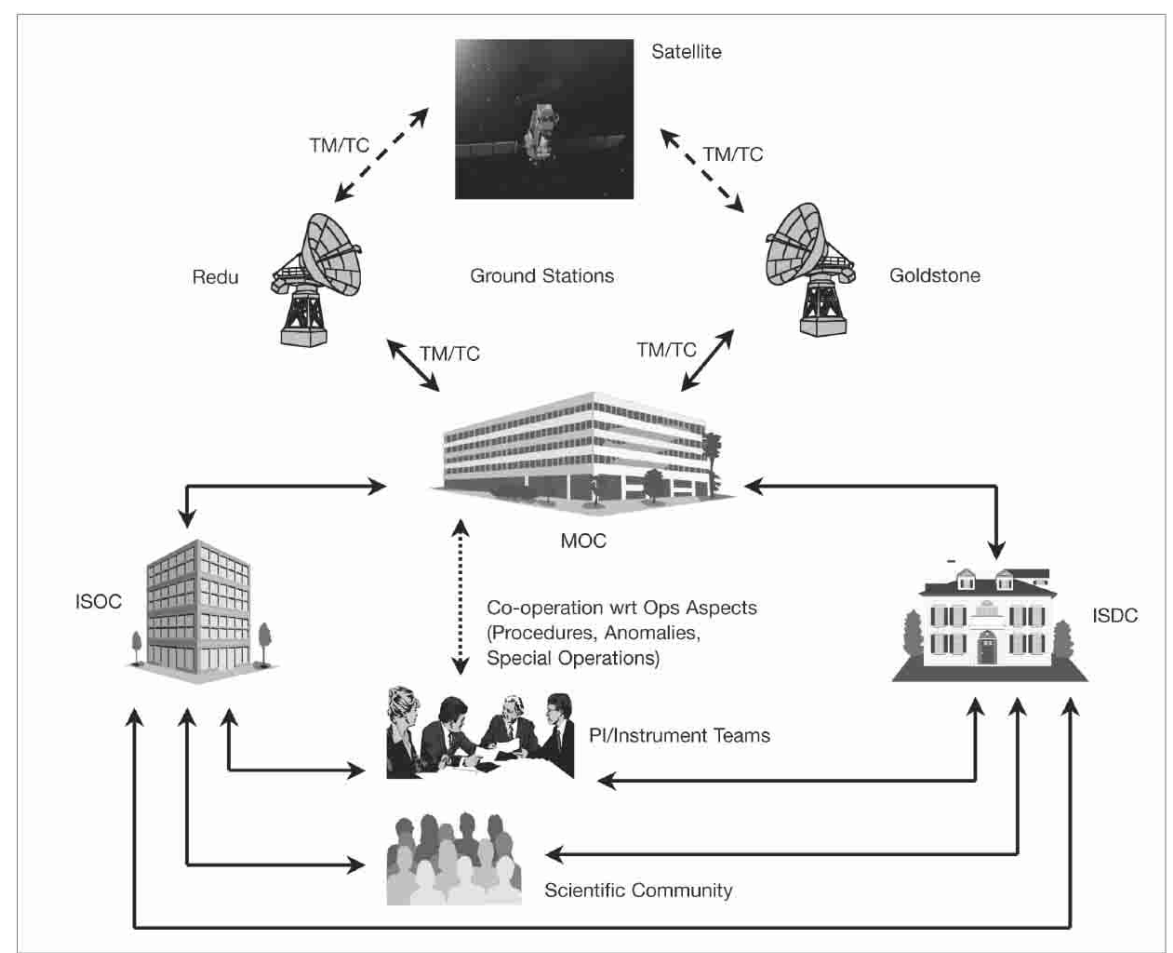

Fig. 1. The overall structure of the INTEGRAL ground segment consisting of the Operational Ground Segment (MOC, ground stations and communication lines) and the Science Ground Segment (ISOC and ISDC).

telemetry from the ground stations. Based on spacecraft and instrument housekeeping data real-time monitoring of the operation and of the safety and health aspects of the spacecraft and instruments are performed at the MOC. All telemetry and auxiliary data required to evaluate the scientific data are routed to the ISDC in real-time.

The MOC maintains the onboard software and the operational database and is responsible for the short- and longterm archiving of the telemetry, telecommands and of the auxiliary data. The archive is regularly consolidated (filling of data gaps) with the satellite playback telemetry received from the ground stations after each pass. The consolidated data are written to CDs within days of a particular orbit's completion and are sent to the ISDC.

\section{The INTEGRAL science ground segment}

The science ground segment consists of the ISDC and the ISOC. Both elements are in contact with the scientific community. The ISOC is mainly involved in the uplink part of the system and the ISDC with the downlink part.

\subsection{The INTEGRAL science operations centre}

The ISOC tasks can be summarized as follows:

- to prepare the Announcements of Opportunity for observations, receive proposals, assess their technical feasibility and to make these assessments available to an INTEGRAL Time Allocation Committee;
- to assume the overall responsibility for the mission planning (scheduling) and for the implementation of the observing programme;

- to coordinate and schedule payload engineering and calibration observations;

- to assume the responsibility for the definition of scientific operations including the instrument configuration for each observation;

- to decide on the generation of an alert for Targets of Opportunity in order to change/interrupt the observing programme (responsibility of the Project Scientist);

- to operate the INTEGRAL help-desk in collaboration with the ISDC;

- to maintain an archive of all scientific data as created and populated by the ISDC.

The ISOC issues the Announcements of Opportunity for observing time. The proposals are submitted to ISOC using the Proposal Generation Tool developed at ISOC. The received proposals are stored in the central ISOC database. The proposals are technically evaluated by ISOC staff for visibility and for adequate observation duration using the Target Visibility Tool and the Observation Time Estimator. Both tools are also accessible to the science community when writing the proposals. All proposals for observing time are assessed and rated by an independent Time Allocation Committee. The accepted proposals are then processed at the ISOC into an optimized observing plan, consisting of a sequence of target positions together with the corresponding instrument configurations. As part of this optimization process the ISOC checks for targets close together in the sky which can be 


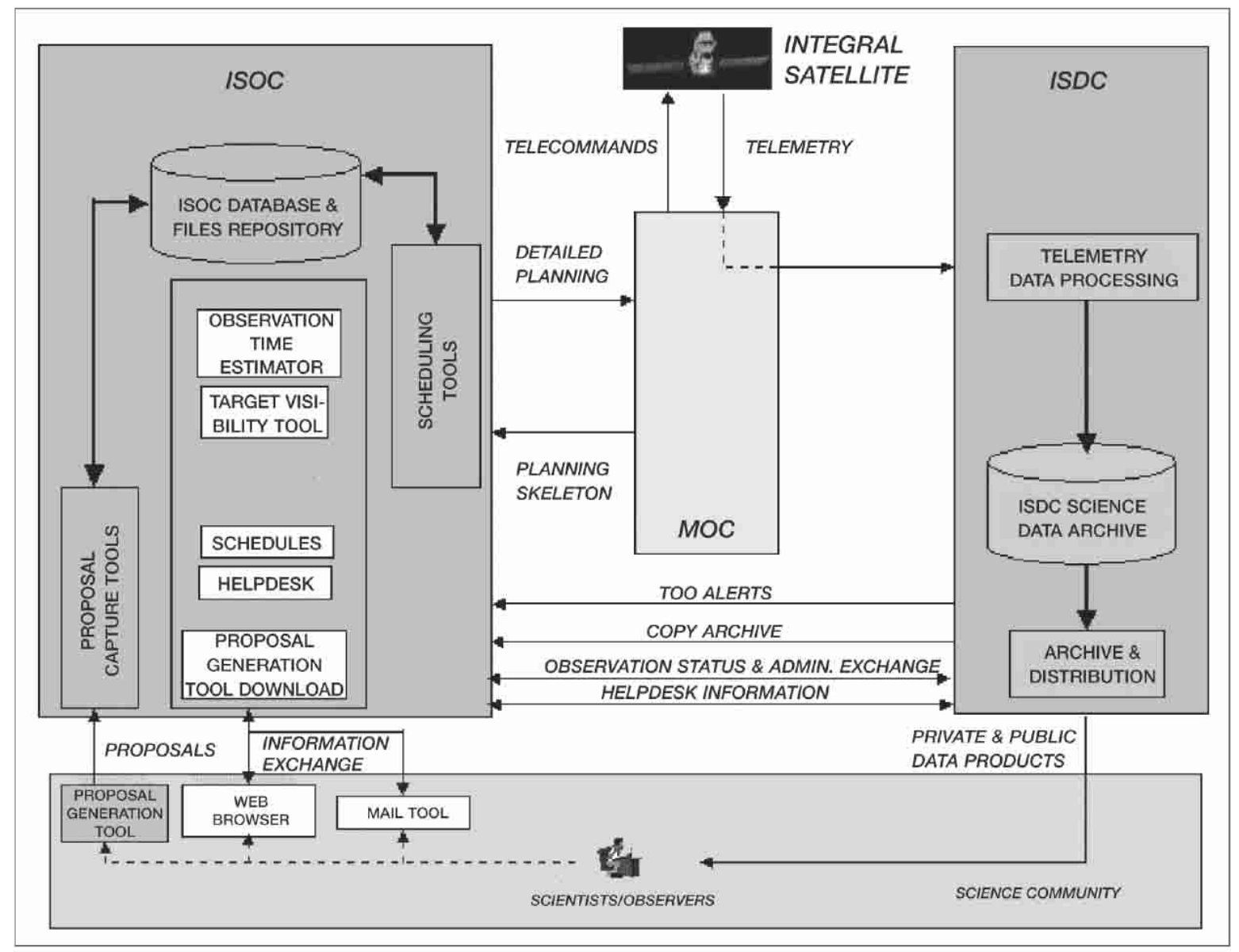

Fig. 2. Data flow in the INTEGRAL Ground Segment. The ISOC and ISDC have direct contact with the scientific community. The MOC is in contact with the INTEGRAL satellite via the ground stations in Goldstone and Redu.

observed in a single observation - so saving observing time. This is particularly important for INTEGRAL, where the observations are generally long and the fields of view of the $\gamma$-ray instruments are large. The optimized observing plan sequences are forwarded to the MOC for creation of the corresponding timelines. Both a long-term and a detailed shortterm schedule are made available on the ISOC Web page (http://www.rssd.esa.int/Integral). The ISOC routinely receives feedback from the ISDC on the status of the executed observations. In case of fully (or partly) unsuccessful execution, rescheduling of the observation is considered.

The ISOC stays in close contact with the instrument teams and the MOC in order to coordinate any changes to the instrument configurations and to plan, coordinate, and schedule payload engineering (e.g. SPI annealing) and calibration observations (e.g. OMC flat field sequences).

A Target of Opportunity (ToO) Observation requires special treatment by the ground segment. A ToO may be discovered using INTEGRAL itself, or by other satellites or groundbased observatories. Requests for a ToO observation can be made by either the ISDC, or the astronomers who made the discovery, using the dedicated INTEGRAL ToO Alert Web page. The Project Scientist then decides on the basis of scientific merit whether to proceed with the ToO request. If the request is granted, the already planned sequence of INTEGRAL observations will be interrupted within 20 to 36 hours and the ISOC will generate a new observing sequence, which will be forwarded to the MOC for validation and execution.

There is one central help-desk dealing with all questions to the INTEGRAL mission. The help-desk is operated jointly by ISOC and ISDC staff. Questions on proposals, observing modes, scheduling and on INTEGRAL in general are handled by ISOC staff and questions on INTEGRAL data, instrument calibration and data shipment are handled by ISDC staff. This split is transparent to the user of the help desk.

The ISOC also maintains a mirror of the scientific data archive populated by the ISDC. This archive is currently restricted to internal ISOC use and it is not foreseen that it will be accessible by the outside world. It does, however, also act as a backup of the ISDC archive. It would become fully operational in case of an anomaly where the ISDC archive is off-line for an extended time period. The ISOC archive guarantees that ESA can satisfy its obligation to ensure the long-term availability of the INTEGRAL data.

The smooth operation of INTEGRAL is reflected in the steady progress of the observation programme. Despite the execution of additonal PV observation in the period from December 17 to 30,2002 and the long period spent on the 
essential Crab calibration (from February 5 to 27, 2003) already 44 individual $\mathrm{AO}-1$ observations were observed up to May 16, 2003, i.e. until five month after the end of the commissioning phase. 18 out of these 44 observations were ToO observations and required a deviation from the long term observation plan. $50 \%$ of the scheduled AO- 1 time was associated with fixed time observations. The expected number of 19 Galactic Plane Scans were executed and $46 \%$ of the Galactic Center Deep Exposure time was already spent. In total $4.8 \times 10^{6} \mathrm{~s}$ and $2.8 \times 10^{6} \mathrm{~s}$ were spent on open and core programme time respectively up to mid May 2003. The share of the core programme time on the total observing time $(36.8 \%)$ is already very close to the $35 \%$ it should be at the end of the first year of operation.

The overall efficiency of the INTEGRAL operation is excellent and higher than was expected before launch. The current scheduling efficiency is $93 \%$, i.e. on average $93 \%$ of the time outside the radiation belts is used for science observations, the remaining time is spent on slews or reaction wheel biases.

\subsection{The INTEGRAL science data centre}

The ISDC is responsible for searching for gamma-ray bursts and other phenomena in the real-time telemetry, for scientific processing of the satellite telemetry, for the archiving of the mission's scientific products and the distribution of the data to the science community. A detailed description of the ISDC is given by Courvoisier et al. (2003).

\section{Summary}

The INTEGRAL ground segment is distributed over three different main sites with well defined tasks. Operations have proceeded remarkably smoothly since launch with $>95 \%$ of the planned operations being executed (with an average of 97\%) and $>95 \%$ of real-time telemetry being received by the ISDC. The good performance of the ground segment is contributing to the overall scientific success of the mission and has validated the design of the overall system and has confirmed its robustness. It demonstrates that the INTEGRAL ground segment is well equipped to fulfill its tasks.

Acknowledgements. The authors wish to thank C. Breneol, N. Dean, F. Jacobs, A. Jeanes, N. Trams, J. Treloar and R. Zondag for their support in the development and operations of the INTEGRAL mission at ISOC.

\section{References}

Courvoisier, T. J.-L., Walter, R., Beckmann, V., et al. 2003, A\&A, 411, L53 